\title{
Spin susceptibility and magnetic short-range order in the Hubbard model
}

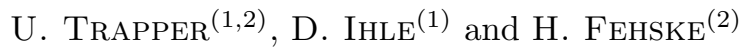 \\ (1) Institut für Theoretische Physik, Universität Leipzig, D-04109 Leipzig, Germany \\ ${ }^{(2)}$ Physikalisches Institut, Universität Bayreuth, D-95440 Bayreuth, Germany
}

(Bayreuth, April 18, 1996)

\begin{abstract}
The uniform static spin susceptibility in the paraphase of the one-band Hubbard model is calculated within a theory of magnetic short-range order (SRO) which extends the four-field slaveboson functional-integral approach by the transformation to an effective Ising model and the selfconsistent incorporation of SRO at the saddle point. This theory describes a transition from the paraphase without SRO for hole dopings $\delta>\delta_{c_{2}}$ to a paraphase with antiferromagnetic SRO for $\delta_{c_{1}}<\delta<\delta_{c_{2}}$. In this region the susceptibility consists of interrelated 'itinerant' and 'local' parts and increases upon doping. The zero-temperature susceptibility exhibits a cusp at $\delta_{c_{2}}$ and reduces to the usual slave-boson result for larger dopings. Using the realistic value of the on-site Coulomb repulsion $U=8 t$ for $\mathrm{La}_{2-\delta} \mathrm{Sr}_{\delta} \mathrm{CuO}_{4}$, the peak position $\left(\delta_{c_{2}}=0.26\right)$ as well as the doping dependence reasonably agree with low-temperature susceptibility experiments showing a maximum at a hole doping of about $25 \%$.
\end{abstract}

PACS number(s): 75.10.-b, 71.28. + d, 71.45.-d

Among the most striking features of high- $T_{c}$ superconductors in the normal state, the unconventional magnetic properties have attracted increasing attention [1]. As revealed by neutron scattering [2] and nuclear magnetic resonance [3] experiments, in the metallic state there exist pronounced antiferromagnetic (AFM) spin correlations which are ascribed to strong Coulomb correlations within the $\mathrm{CuO}_{2}$ planes. Knight-shift [4] and bulk measurements [4 6 ] of the spin susceptibility $\chi(T, \delta)$ in $\mathrm{La}_{2-} \mathrm{Sr}_{\delta} \mathrm{CuO}_{4}$ show a maximum in the doping dependence as well as, for moderate hole doping $(\delta \leq 0.21)$, in the temperature dependence, where the temperature of the maximum decreases with increasing doping. Such a behaviour, also observed in $\mathrm{YBa}_{2} \mathrm{Cu}_{3} \mathrm{O}_{6+\mathrm{y}}(y \leq 0.92)$ [7, 8], may be qualitatively understood as an effect of AFM short-range order (SRO) which decreases with increasing doping and temperature.

Up to now there have been only a few attempts, based on one-band [9 12] and three-band [13] correlation models, to describe the unusual doping and temperature dependence of the normal-state susceptibility. In the $t-t^{\prime}-\mathrm{J}$ model, a maximum in $\chi$ was obtained for the Pauli susceptibility of a strongly renormalized quasiparticle band [9] or for the RPA slave-boson susceptibility [10] showing a cusp in the temperature dependence at the transition to the singlet RVB state. In the one-band Hubbard model, a maximum in the doping dependence of $\chi$ was found by a semi-phenomenological weak-coupling approach [11] or by the composite operator method [12]. The role played by SRO in explaining the normal-state susceptibility was investigated on the basis of the three-band Hubbard model 13] by means of a slave-boson CPA theory which, however, is self-consistent only at the single-site level and does not hold at very low temperatures. To improve the treatment of SRO in the paraphase being valid also at $T=0$, in a previous communication [14], hereafter referred to as I, we have presented the main features of a theory of magnetic SRO in the one-band Hubbard model based on the scalar four-field slave-boson (SB) approach 15]. In I we have focused on the stability of magnetic long-range order (LRO) versus SRO, where magnetic LRO phases are found to make way to a paraphase with $\mathrm{SRO}$ in a wide doping region.

In this Brief Report we extend our theory by the inclusion of an external magnetic field $h$ and by the calculation of the uniform static spin susceptibility $\chi$ in the paraphase, where special care is taken to the influence of SRO.

Following the lines indicated in I, the action of the SB functional integral for the partition function of the 2D Hubbard model is expressed in terms of the SB fields $m_{i}, \xi_{i}, n_{i}, \nu_{i}, d_{i}$ and $d_{i}^{*}$ [16. To treat the fluctuations of the local magnetizations $m_{i}$ and the internal magnetic fields $\xi_{i}$ we write $m_{i}=\bar{m}_{i} s_{i}, \xi_{i}=\bar{\xi}_{i} s_{i}\left(s_{i}= \pm\right)$ and make the ansatz $b_{i} \rightarrow b_{s_{i}}$ for the magnetic amplitudes $b \in\{\bar{m}, \bar{\xi}\}$ and the charge degrees of freedom $b \in\left\{n, \nu, d=d^{*}\right\}$. We transform the free-energy functional $\Psi$ to an effective Ising model in the nearest-neighbour pair $(\langle i j\rangle)$ approximation and obtain

$$
\Psi\left(\left\{s_{i}\right\}\right)=\bar{\Psi}-\bar{h} \sum_{i} s_{i}-\bar{J} \sum_{\langle i j\rangle} s_{i} s_{j}
$$


with

$$
\begin{aligned}
\bar{\Psi}= & -\frac{1}{\beta} \sum_{\vec{k} \sigma} \ln \left[1+\exp \left\{-\beta\left[\left(z_{\sigma}^{o}\right)^{2} \varepsilon_{\vec{k}}+\nu^{o}-\sigma\left(\xi^{o}+h\right)-\mu\right]\right\}\right] \\
& +\frac{N}{2} \sum_{\alpha= \pm 1}\left\{U d_{\alpha}^{2}-n_{\alpha} \nu_{\alpha}+\bar{m}_{\alpha} \bar{\xi}_{\alpha}+\sum_{\sigma}\left(\Phi_{\alpha \sigma}+\Phi_{\alpha \alpha \sigma}+\Phi_{-\alpha \alpha \sigma}\right)\right\}, \\
\bar{h}= & -\frac{1}{2} \sum_{\alpha} \alpha\left[U d_{\alpha}^{2}-n_{\alpha} \nu_{\alpha}+\bar{m}_{\alpha} \bar{\xi}_{\alpha}+\sum_{\sigma}\left(\Phi_{\alpha \sigma}+2 \Phi_{\alpha \alpha \sigma}\right)\right], \\
\bar{J}= & -\frac{1}{4} \sum_{\alpha \sigma}\left(\Phi_{\alpha \alpha \sigma}-\Phi_{-\alpha \alpha \sigma}\right) .
\end{aligned}
$$

The single-site and two-site fluctuation contributions $\Phi_{\alpha \sigma}=\left.\Phi_{i \sigma}\left(\alpha_{i}\right)\right|_{\alpha_{i}=\alpha}$ and $\Phi_{\alpha \alpha^{\prime} \sigma}=\left.\Phi_{\langle i j\rangle \sigma}\left(\alpha_{i}, \alpha_{j}\right)\right|_{\substack{\alpha_{i}=\alpha \\ \alpha_{j}=\alpha^{\prime}}}$, respectively, are given by

$$
\begin{aligned}
\Phi_{i \sigma} & =\frac{1}{\pi} \int d \omega f(\omega-\mu) \operatorname{Im} \ln \left[1-G_{i i \sigma}^{o} V_{i \sigma}\left(\alpha_{i}\right)\right], \\
\Phi_{\langle i j\rangle \sigma} & =\frac{1}{\pi} \int d \omega f(\omega-\mu) \operatorname{Im} \ln \left[1-G_{\langle i j\rangle \sigma}^{o} T_{j \sigma}\left(\alpha_{j}\right) G_{\langle j i\rangle \sigma}^{o} T_{i \sigma}\left(\alpha_{i}\right)\right] .
\end{aligned}
$$

In (6), $G_{i j \sigma}^{o}(\omega)$ is the uniform paramagnetic $(\mathrm{PM})$ Green propagator, and the scattering matrix $T_{i \sigma}=$ $V_{i \sigma}\left(1-G_{i i \sigma}^{o} V_{i \sigma}\right)^{-1}$ is expressed in terms of the local perturbation

$$
V_{i \sigma}\left(\alpha_{i}, \omega\right)=\frac{1}{\left(z_{\alpha_{i} \sigma}\right)^{2}}\left\{\left[\left(z_{\alpha_{i} \sigma}\right)^{2}-\left(z_{\sigma}^{o}\right)^{2}\right]\left[\omega-\nu^{o}+\sigma\left(\xi^{o}+h\right)\right]+\left(z_{\sigma}^{o}\right)^{2}\left[\nu_{\alpha_{i}}-\nu^{o}-\sigma\left(\alpha_{i} \bar{\xi}_{\alpha_{i}}-\xi^{o}\right)\right]\right\},
$$

where

$$
z_{\alpha_{i} \sigma}=\frac{\sqrt{2}\left[\sqrt{\left(n_{\alpha_{i}}+\sigma \alpha_{i} \bar{m}_{\alpha_{i}}-2 d_{\alpha_{i}}^{2}\right)\left(1-n_{\alpha_{i}}+d_{\alpha_{i}}^{2}\right)}+d_{\alpha_{i}} \sqrt{n_{\alpha_{i}}-\sigma \alpha_{i} \bar{m}_{\alpha_{i}}-2 d_{\alpha_{i}}^{2}}\right]}{\sqrt{\left(n_{\alpha_{i}}+\sigma \alpha_{i} \bar{m}_{\alpha_{i}}\right)\left(2-n_{\alpha_{i}}-\sigma \alpha_{i} \bar{m}_{\alpha_{i}}\right)}}
$$

and the superscript " $o$ " refers to PM saddle-point values. By the functional (1) we determine the saddle point for all Bose fields $b_{\alpha}=\left\{\bar{m}_{\alpha}, \bar{\xi}_{\alpha}, n_{\alpha}, \nu_{\alpha}, d_{\alpha}\right\}$ in the external field $h$, where, in the spirit of I, the SRO is self-consistently incorporated within the Bethe cluster approximation (taking into account only the nearest-neighbour SRO). As found in I, in the $h=0$ limit $\left(\bar{m}_{\alpha}=\bar{m}\right)$ the self-consistent calculation of the effective Ising-exchange integral $\bar{J}$ as function of the interaction strength $U$ and the hole doping $\delta=1-n$ yields two possible paraphases $\left(\left\langle s_{i}\right\rangle=0\right)$ : (i) the paraphase without SRO (PM; $\bar{J}=0, \bar{m}=0$ ) and (ii) the paraphase with antiferromagnetic SRO (SRO-PM; $\bar{J}<0, \bar{m}>0$ ).

The uniform static spin susceptibility $\chi(T, \delta)$ has to be calculated according to

$$
\chi=\lim _{h \rightarrow 0} \sum_{\alpha}\left(W_{\alpha} \frac{d m_{\alpha}}{d h}+m_{\alpha} \frac{d W_{\alpha}}{d h}\right)
$$

where $m_{\alpha}=\bar{m}_{\alpha} \alpha, W_{\alpha}=W_{\alpha}\left(\bar{h}, h^{*}, \bar{J}\right)$ is the probability for the Ising spin $\alpha$ at the central site of the Bethe cluster, and $h^{*}$ is the effective Bethe field. The first term in (9) describes the change of the magnetization-amplitude with the applied magnetic field and gives mainly the 'itinerant' contribution to $\chi$. The second term describes directional fluctuations of the local magnetizations and is called the 'local' contribution being finite only in the SRO-PM phase. Note that the 'itinerant' and 'local' properties are interrelated and determine both contributions to the spin susceptibility. In the PM and SRO-PM phases we have calculated the doping dependence of the zero-temperature susceptibility in the 2D Hubbard model (being finite in contrast to the theory of Ref. [13]) in a completely self-consistent way, where in the tedious numerical evaluation of the integrals (5) and (6) and of their derivatives particular attention has to be paid to the analytical behaviour of the complex logarithm. 


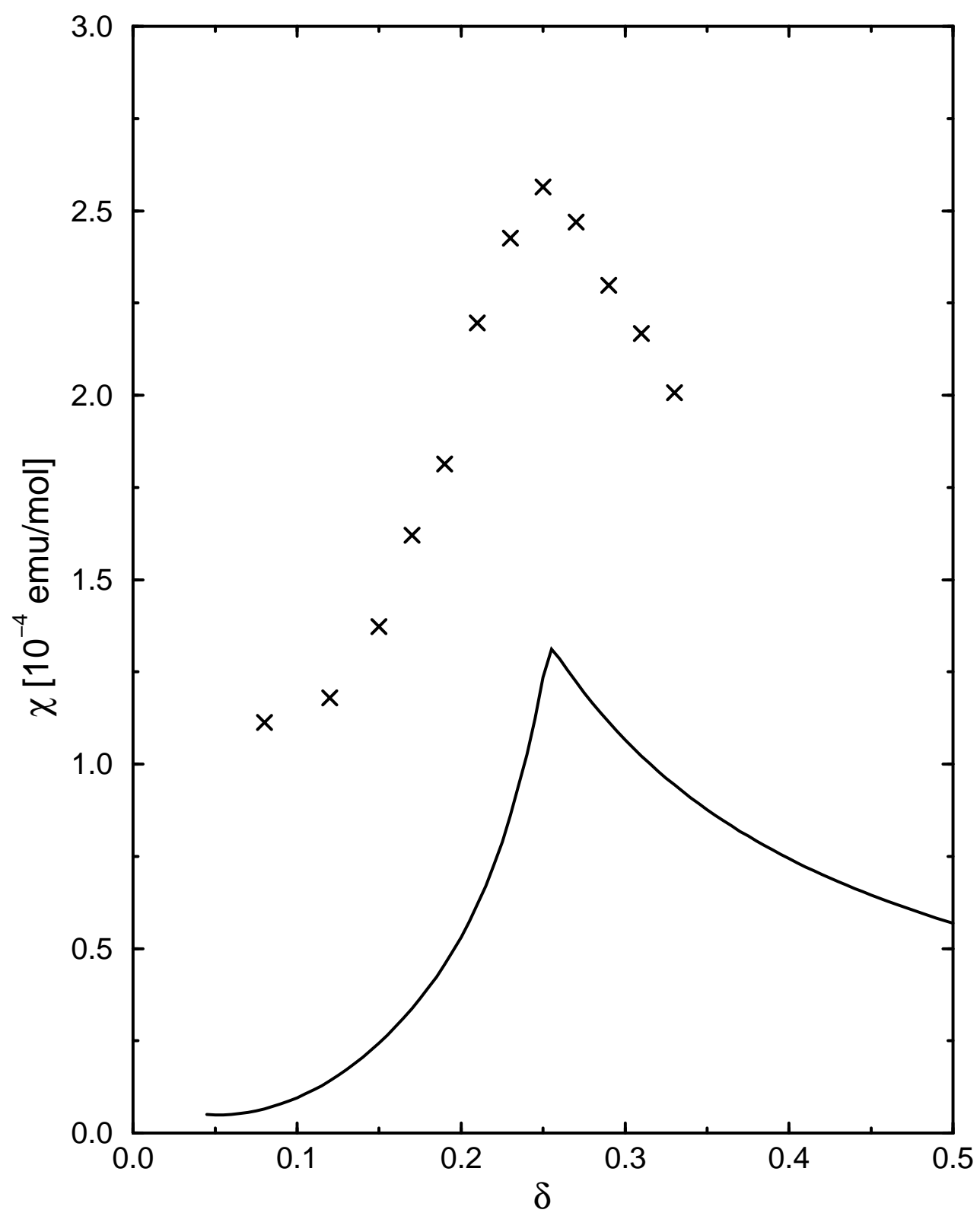

FIG. 1. Uniform static spin susceptibility as a function of doping at $T=0$. The theoretical result obtained for the $2 \mathrm{D}$ Hubbard model at $U / t=8$ and $t=0.3 \mathrm{eV}$ (solid) is compared with the spin contribution $(\times)$ to the (corrected) experimental susceptibility on $\mathrm{La}_{2-\delta} \mathrm{Sr}_{\delta} \mathrm{CuO}_{4}$ at $T=50 \mathrm{~K}$ 递, 6 .

Figure 1 shows our result without any fit procedure using the commonly accepted value $U / t=8$ for the Hubbard model applied to high- $T_{c}$ cuprates [17]. As stated in I, in the region $6<U / t<12$, there occurs a first-order $(1,1)$-spiral $\rightleftharpoons \mathrm{SRO}-\mathrm{PM}$ transition at $\delta_{c_{1}}$ and a SRO-PM $\rightleftharpoons \mathrm{PM}$ transition of second order at $\delta_{c_{2}}$. In the PM phase $\left(\delta>\delta_{c_{2}}\right)$ the SB band-renormalized Pauli susceptibility has a pronounced doping dependence in two dimensions and agrees with the static and uniform limit of the dynamic spin susceptibility derived, within the spin-rotationinvariant SB scheme [18], from the Gaussian fluctuation matrix at the PM saddle point [19]. In the SRO-PM phase $\left(\delta_{c_{1}}<\delta<\delta_{c_{2}}\right)$, the Pauli susceptibility is suppressed due to the SRO-induced spin stiffness against the orientation of the local magnetizations along the homogeneous external field. Accordingly, at $\delta_{c_{2}}$ a cusp in $\chi(0, \delta)$ appears. Since, for $\delta_{c_{1}}<\delta<\delta_{c_{2}},|\bar{J}|$ decreases with increasing $\delta$ [14], the susceptibility increases upon doping.

The peak in $\chi(0, \delta)$ only appears at sufficiently high ratios $U / t>6$, for which a $\mathrm{SRO}-\mathrm{PM} \rightleftharpoons \mathrm{PM}$ transition may occur. According to the phase diagram, given in Fig. 2 of I, in the region $6<U / t<12$ the SRO-PM $\rightleftharpoons$ PM transition shifts to higher doping values with increasing $U / t$. Correspondingly, the peak position in $\chi(0, \delta)$ reveals the same $U / t$ dependence.

In Fig. 1 we have also depicted the spin contribution to the magnetic susceptibility of $\mathrm{La}_{2-\delta} \mathrm{Sr}_{\delta} \mathrm{CuO}_{4}$ at $50 \mathrm{~K}$ 
obtained from the experimental data on the total susceptibility [5] by subtracting the diamagnetic core $(-9.9 \times$ $\left.10^{-5} \mathrm{emu} / \mathrm{mol}\right)$ and Van Vleck $\left(2.4 \times 10^{-5} \mathrm{emu} / \mathrm{mol}\right)$ contributions which, according to Ref. [6], can be taken to be independent of doping and temperature over the limited parameter region studied here. As Fig. 1 shows, the experimentally observed pronounced maximum at a hole doping of about $25 \%$ is reproduced very well by our theory yielding the peak position at $\delta_{c_{2}}=0.26(U / t=8)$. Moreover, the qualitative doping dependence of $\chi$ reasonably agrees with experiments. Of course, it could not be expected that our approach based on the simple (single-band) Hubbard model yields the correct magnitude of $\chi$ for $\mathrm{La}_{2-\delta} \mathrm{Sr}_{\delta} \mathrm{CuO}_{4}$. Especially, concerning the low-doping limit $\delta \rightarrow \delta_{c_{1}}=0.04$, the theoretical susceptibility is much too low as compared with experiments. This deficiency may be explained as follows. For $\delta=0$ and large $U / t$ values, the Hubbard model is equivalent to the Heisenberg antiferromagnet with the exchange interaction $J=4 t^{2} / U$. In this model, the spin susceptibility at $T=0$ has a finite value proportional to $J^{-1}$ [20] which is due to the existence of transverse spin fluctuations. However, our scalar four-field SB approach to the spin susceptibility in the presence of SRO implies the transformation of the free-energy functional to an effective Ising model describing longitudinal fluctuations only. Since the 'local' contribution to $\chi$ is of Ising-type, we get a too small susceptibility in the low-doping limit which, however, is finite due to the interrelation to the 'itinerant' contribution to $\chi$. Therefore, we suggest that a theory of SRO based on the spin-rotation-invariant SB scheme [18] and resulting in an effective Heisenberg-model functional may improve the results in the magnitude of $\chi$, in particular at low doping levels.

Finally, we notice that the increase of the susceptibility upon doping obtained within our theory for moderate Coulomb repulsions $(U / t>6)$ is in qualitative accord with recent QMC data [21] and with the approaches of Refs. [11] and [12]. However, in those works a maximum in the spin susceptibility was found even at a smaller coupling $(U / t=4)$.

From our results we conclude that the concept of magnetic SRO in strong-correlation models may play the key role in the explanation of many unconventional properties of high- $T_{c}$ compounds. The theory may be extended in several directions. As discussed above a spin-rotation-invariant theory of SRO may improve the agreement of the spin susceptibility with experiments. Furthermore, as motivated by neutron scattering experiments [2] probing the AFM correlation length over several lattice spacings, the effects of a longer than nearest-neighbour ranged SRO (which may be described beyond the nearest-neighbour pair approximation) should be investigated.

This work was performed under the auspices of Deutsche Forschungsgemeinschaft under project SF-HTSL-SRO. H.F. acknowledges the support of the NTZ and the hospitality at the University of Leipzig.

[1] A. P. Kampf, Physics Reports 249, 219 (1994).

[2] J. Rossat-Mignod et al., Physica B 186-188, 1 (1993).

[3] M. Horvatic et al., Physica C 166, 151 (1990).

[4] Y.-Q. Song, M. A. Kennard, K. R. Poeppelmeier, and W. P. Halperin, Phys. Rev. Lett. 70, 3131 (1993).

[5] J. B. Torrance et al., Phys. Rev. B 40, 8872 (1989).

[6] D. C. Johnston, Phys. Rev. Lett. 62, 957 (1989).

[7] Z. P. Han and R. Dupree, Physica C 208, 328 (1993).

[8] R. E. Walstedt et al., Phys. Rev. B 41, 9574 (1990); B 45, 8047 (1992).

[9] S. A. Trugman, Phys. Rev. Lett. 65, 500 (1990).

[10] T. Tanamoto, K. Kuboki, and H. Fukuyama, J. Phys. Soc. Jpn. 60, 3072 (1991); T. Tanamoto, H. Kohno, and H. Fukuyama, J. Phys. Soc. Jpn. 62, 717 (1993); 62, 1455 (1993).

[11] T. Kopp and F. Mila, Phys. Rev. B 43, 12980 (1991); 50, 13017 (1994).

[12] F. Mancini, S. Marra, and H. Matsumoto, Physica C 252, 361 (1995).

[13] G. Baumgärtel, J. Schmalian, and K. H. Bennemann, Europhys. Lett. 24, 601 (1993).

[14] U. Trapper, D. Ihle, and H. Fehske, Phys. Rev. B 52, R11553 (1995).

[15] G. Kotliar and A. E. Ruckenstein, Phys. Rev. Lett. 57, 1362 (1986).

[16] H. Hasegawa, J. Phys. Condens. Matter 1, 9325 (1989).

[17] M. S. Hybertsen, E. B. Stechel, M. Schlüter, and D. R. Jennison, Phys. Rev. B 41, 11068 (1990); M. S. Hybertsen, E. B. Stechel, W. M. C. Foulkes, and M. Schlüter, Phys. Rev. B 45, 10032 (1992).

[18] T. Li, P. Wölfle, and P. J. Hirschfeld, Phys. Rev. B 40, 6817 (1989).

[19] T. Li, Y. S. Sun, and P. Wölfle, Z. Phys. B 82, 369 (1991).

[20] T. Barnes, Int. J. Mod. Phys. C 2, 659 (1991).

[21] L. Chen and A.-M. S. Tremblay, Phys. Rev. B 49, 4338 (1994). 